\title{
miR-I306 Promotes Lung Squamous Cell Carcinoma Progression and Predicts Clinical Prognosis of Patients
}

Mei $\mathrm{Li}^{1}, *$

Chunxiang $\mathrm{Xu}^{2, *}$

Yan Wang ${ }^{3}$

Hua $\mathrm{Liu}^{4}$

'Department of Chemotherapy, Affiliated Hospital of Nantong University, Nantong, Jiangsu, People's Republic of China;

${ }^{2}$ Department of Orthopedics, Affiliated Hospital of Nantong University, Nantong, Jiangsu, People's Republic of China; ${ }^{3}$ Department of Radiotherapy, Affiliated Hospital of Nantong University, Nantong, Jiangsu, People's Republic of China; ${ }^{4}$ Department of Respiratory, Affiliated Hospital of Nantong University, Nantong, Jiangsu, People's Republic of China

*These authors contributed equally to this work
Correspondence: Hua Liu

Department of Respiratory, Affiliated Hospital of Nantong University, 20 Xisi

Road, Nantong, Jiangsu, 22600I, People's

Republic of China

$\mathrm{Tel} / \mathrm{Fax}+86-5$ I 3-85052222

Email hua5309lao@I63.com

Yan Wang

Department of Radiotherapy, Affiliated

Hospital of Nantong University, 20 Xisi

Road, Nantong, Jiangsu, 22600I, People's

Republic of China

$\mathrm{Tel} / \mathrm{Fax}+86-513-85052222$

Email yan9963I8lan@I63.com
Purpose: Lung squamous cell carcinoma (LUSC) is one of the major subtypes of non-small cell lung cancer (NSCLC) with high mortality. Identification of novel biomarkers of the development of LUSC could provide basis for clinical treatment and improve patients' prognosis. The function of miR-1306 in the development of LUSC was investigated.

Patients and Methods: A total of 103 paired LUSC and normal tissues were collected from LUSC patients. The expression of miR-1306 in collected tissues and cultured cells was evaluated by PCR. The clinical significance of miR-1306 was assessed by a series of statistical analyses, and the biological effect of miR-1306 was also estimated with the CCK8 and Transwell assay.

Results: The significant upregulation of miR-1306 was observed in LUSC, which was associated with positive lymph node metastasis and advanced TNM stage of patients. miR1306 was also identified as an independent prognostic factor negatively associated with the prognosis of patients. Additionally, the upregulation of miR-1306 was found to promote the proliferation, migration, and invasion of LUSC cells, indicating its tumor enhancer role in the development of LUSC. While miR-1306 was also found to regulate RBM3, which was speculated to be the mechanism underlying the function of miR-1306.

Conclusion: miR-1306 functions as a prognostic indicator and tumor promoter of LUSC through targeting RBM3, which provides a potential therapeutic target of LUSC.

Keywords: miR-1306, lung squamous cell carcinoma, prognosis, disease development, cell proliferation, migration, invasion

\section{Introduction}

Non-small cell lung cancer (NSCLC) accounts for a majority cases of lung cancer, which remains one of the leading causes of cancer-related death worldwide also in China. ${ }^{1,2}$ Among the histological types of NSCLC, lung squamous cell carcinoma (LUSC) accounts for approximately one-third of NSCLC cases. ${ }^{3,4}$ Although great progress has been made in the therapeutic strategy of LUSC, the clinical outcome of LUSC is still unsatisfied. ${ }^{5}$ Exploring more novel and efficient biomarkers for the early detection and risk prediction of LUSC is of great significance, which can improve clinical prognosis and postoperative quality of life in patients.

microRNAs (miRNAs) are short noncoding RNAs regulating the expression of target gene and the translation of proteins via mediating the stability and translation of mRNAs. ${ }^{6}$ Emerging evidence demonstrated miRNAs contributed to the tumorigenesis, development, and metastasis of various cancers, including NSCLC and 
LUSC. ${ }^{7,8}$ For example, miR-486-5p has been revealed to be an antioncogene of LUSC and possessed the function of LUSC diagnosis. ${ }^{9}$ MiR192 and miR-622 could suppress the sensitivity of LUSC cells to the chemotherapy and inhibit the colony formation of motility. ${ }^{10}$ Using miRNA as biomarkers in cancer research could enable the early detection of malignant tumors and improve the therapeutic options of patients. ${ }^{11}$ The identification of novel efficient biomarkers is of great significance in the clinical management of LUSC.

miR-1306 is a novel member of miRNA family, which has been demonstrated to be dysregulated and act critical roles in various human diseases. The upregulation miR1306 in heart failure patients and showed dramatical protective effect on the cerebral ischemia/reperfusion injury. ${ }^{12}$ In hepatocellular cell carcinoma, miR-1306 promoted cell migration and invasion in vitro and in vivo. ${ }^{13}$ Previous identification of differently expression miRNA in NSCLC revealed the significant upregulation of miR-1306 in all subtypes of NSCLC. ${ }^{14}$ The specific performance of miR1306 in LUSC remains to be disclosed.

The present study aims to investigate the expression level of miR-1306 in LUSC and evaluate its specific significance and function in patients' outcome and disease development.

\section{Materials and Methods}

\section{Patients and Samples}

This study had obtained approval from the Ethics Committee of Affiliated Hospital of Nantong University and was in accordance with the Declaration of Helsinki. One hundred and three LUSC patients were included in this study from January 2012 to December 2015 at Affiliated Hospital of Nantong University. The recruited patients had never received any types of chemotherapy or radiotherapy before surgery. The tumor tissues and matched normal tissues were collected during surgery resection and stored at $-80^{\circ} \mathrm{C}$. The patients were followed up for 5 years after their surgeries to track their survival status.

\section{Real-Time qPCR}

Collected tissues were lysed and isolated total RNA with TRIZOL (Invitrogen, USA) according to the protocols. cDNA was generated with the miScript reverse transcription kit (Qiagen) and PCR was executed with the SYBR PrimeScript TM miRNA RT-PCT kit (Takara). Cel-miR
-39 was used as the internal standard to assess the expression level of miR-1306 with the $2^{-\Delta \Delta \mathrm{CT}}$ method.

\section{Cell Culture and Cell Transfection}

LUSC cell lines (NCI-H1703, NCI-H2170, NCI-H520, SKMES-1) and the normal BEAS-2B cells were purchased from ATCC. Cells were cultured in the DMEM culture medium (Invitrogen, $\mathrm{USA}$ ) at $37^{\circ} \mathrm{C}$ with $5 \% \mathrm{CO}_{2}$. Cultured cells were transfected with miR-1306 mimic or inhibitor, or their corresponding negative controls with the help of Lipofectamine 3000 Transfection reagent (Invitrogen, USA). The efficiency of cell transfection was evaluated by PCR.

\section{Cell Proliferation Assay}

The transfected cells were seeded into the 96-well plates supplied with DMEM culture medium and incubated for specific period of time $(0,24,48$, and $72 \mathrm{~h})$ at $37^{\circ} \mathrm{C}$ with $5 \%$ $\mathrm{CO}_{2}$. Then, the CCK8 solution was added to each well with the volume of $10 \mu \mathrm{L}$. After incubating for another $2 \mathrm{~h}$, the absorbance at $450 \mathrm{~nm}$ was detected with the microplate reader.

\section{Cell Migration and Invasion Assay}

A total of $5 \times 10^{4}$ cells/well were seeded into the upper of the Transwell chamber (24-well, pore size of $8 \mu \mathrm{m}$, Corning). The serum-free culture medium was supplied in the upper chamber, while the culture medium with $10 \%$ FBS was placed in the bottom chamber. After $24 \mathrm{~h}$ of incubation, the migrated and invaded cells were fixed with paraformaldehyde and stained with crystal violet and counted under a microscope. The Matrigel (Corning, USA) was used to pre-coat the upper chamber in the invasion assay.

\section{Dual-Luciferase Reporter Assay}

The NCI-H2170 cells were co-transfected with the RBM3 wild-type vector or RBM3 mutant-type vector and miR1306 mimic, inhibitor, or negative controls with the help of Lipofectamine 3000 Transfection reagent (Invitrogen, USA). The relative luciferase activity of RBM3 was evaluated with the Dual-Glo Luciferase Assay System E2920 (Promega, USA). The relative luciferase activity of RBM3 was evaluated with Renilla as the internal reference.

\section{Statistical Analysis}

All data were obtained from triplicate experiments and presented as mean value $\pm \mathrm{SD}$. The statistical analysis of the obtained data was performed with SPSS 23.0 Software. The difference between two groups was evaluated with 
Student's $t$-test, and the multiple comparison among diverse groups was conducted with one-way ANOVA. The clinical significance of miR-1306 was estimated with the $\mathrm{Chi}^{2}$ test, Kaplan-Meier, and Cox regression analysis. $P<0.05$ was considered to be statistically significant.

\section{Results}

\section{The Expression Level of miR-I306 in LUSC}

In collected tissues, miR-1306 showed a significant upregulation in LUSC tissues in comparison with matched normal tissues $(P<0.001$, Figure 1A). Consistently, in LUSC cell lines, the expression of miR-1306 was higher than that in normal cells with the average value of 2.03 , and the differences were significant $(P<0.05, P<0.01, P<0.001$, Figure 1B).

\section{The Clinical Significance of miR-I306 in LUSC}

All patients were divided into two groups (low miR-1306 group and high miR-1306 group) based on the median miR-1306 expression. The low miR-1306 group contained 51 patients consist of 33 males and 18 females, while the high miR-1306 group contained 52 patients including 29 males and 23 females. Patients with positive lymph node metastasis (8 low miR-1306 and 19 high miR-1306) and advanced TNM stage (5 low miR-1306 and 17 high miR1306) showed a high expression level of miR-1306. The significant association of miR-1306 expression with the lymph node metastasis status $(P=0.016)$ and the TNM stage $(P=0.005)$ of patients was observed (Table 1$)$.

A

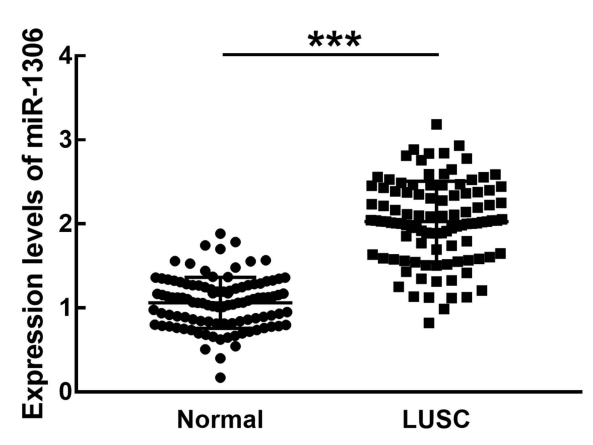

Table I The Association Between miR-I306 Expression and the Clinical Features of Patients

\begin{tabular}{|c|c|c|c|c|}
\hline & \multirow[t]{2}{*}{$\begin{array}{c}\text { Total } n= \\
103\end{array}$} & \multicolumn{2}{|c|}{$\begin{array}{l}\text { miR-I306 } \\
\text { Expression }\end{array}$} & \multirow[t]{2}{*}{$P$ value } \\
\hline & & $\begin{array}{c}\text { Low } \\
(n=5 I)\end{array}$ & $\begin{array}{c}\text { High } \\
(n=52)\end{array}$ & \\
\hline Age & & & & 0.093 \\
\hline$<60$ & 54 & 31 & 23 & \\
\hline$\geq 60$ & 49 & 20 & 29 & \\
\hline Gender & & & & 0.354 \\
\hline Male & 62 & 33 & 29 & \\
\hline Female & $4 I$ & 18 & 23 & \\
\hline Smoking & & & & 0.250 \\
\hline Yes & 65 & 35 & 30 & \\
\hline No & 38 & 16 & 22 & \\
\hline LNM & & & & 0.016 \\
\hline Negative & 76 & 43 & 33 & \\
\hline Positive & 27 & 8 & 19 & \\
\hline TNM stage & & & & 0.005 \\
\hline I-II & 81 & 46 & 35 & \\
\hline III & 22 & 5 & 17 & \\
\hline Vascular invasion & & & & 0.134 \\
\hline Negative & 57 & 32 & 25 & \\
\hline Positive & 46 & 19 & 27 & \\
\hline
\end{tabular}

Abbreviation: LNM, lymph node metastasis.

The difference in patients' survival was assessed by the Kaplan-Meier analysis. The patients with high expression level of miR-1306 showed a worse survival than patients with low miR-1306 expression (log-rank $P=0.034$, Figure 2). Meanwhile, the results of multivariate Cox regression analysis demonstrated miR-1306

B

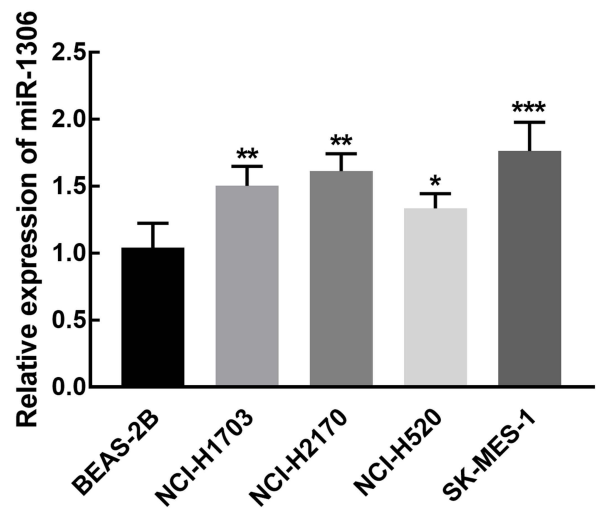

Figure I Expression of miR-I306 in LUSC. (A) The significant upregulation of miR-1306 was observed in LUSC tissues compared with the normal tissues. (B) The expression of miR-1306 in LUSC cells (NCl-HI703, NCl-H2170, NCl-H520, and SK-MES-I cells) was significantly higher than that in the normal cell (BEAS-2B cell). $* P<$ $0.05, * * p<0.01, * * * p<0.001$. 


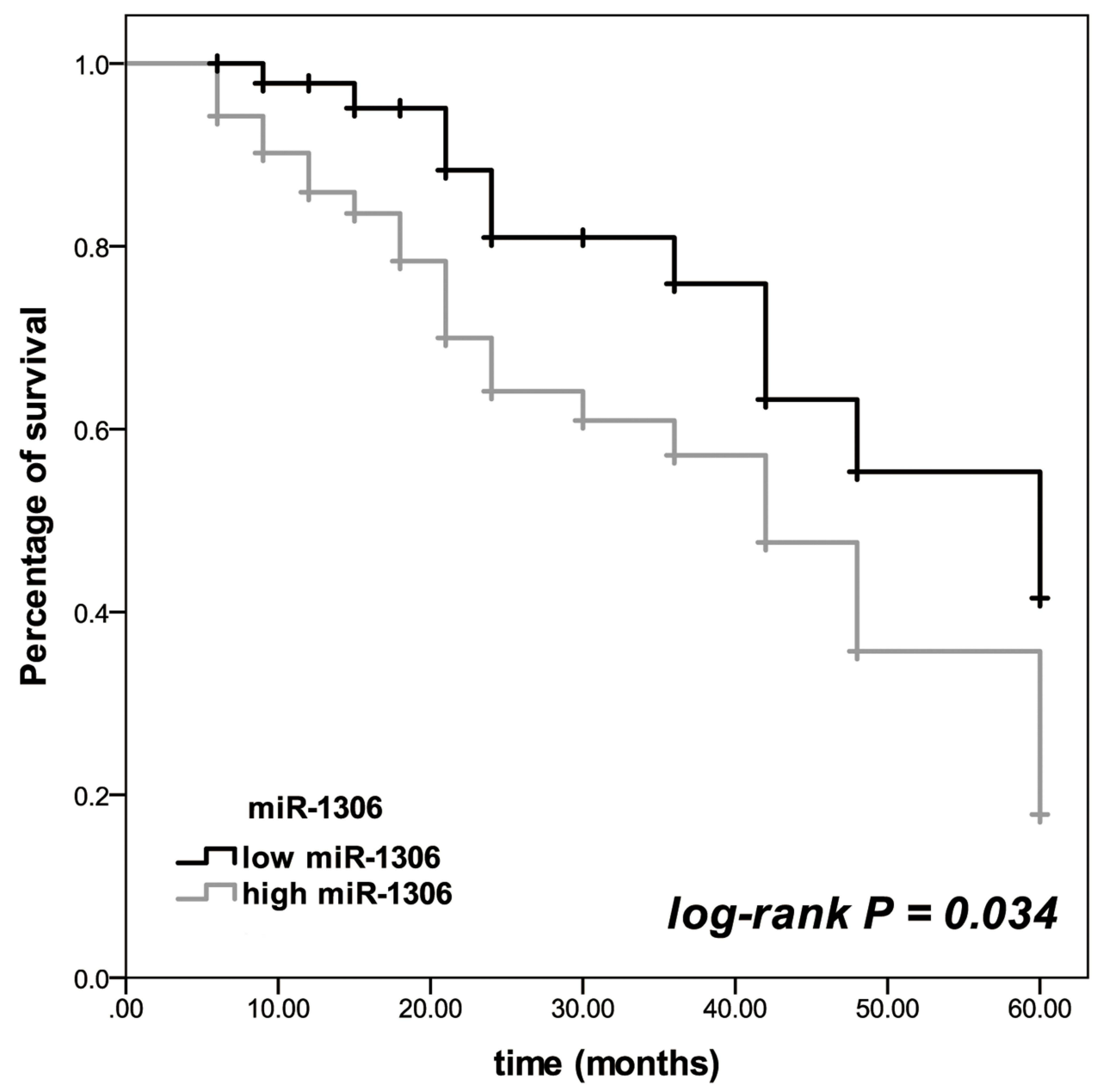

Figure 2 Kaplan-Meier curve of LUSC patients based on the median expression of miR-I306 in LUSC tissues. Patients with relatively high miR-I306 expression showed a significantly poor survival time. Log-rank $P=0.034$.

$(95 \% \mathrm{CI}=1.079-5.444, P=0.032)$ and $\mathrm{TNM}$ stage (95\% CI $=1.060-4.666, P=0.035)$ served as two independent prognostic factors with the HR value of 2.423 and 2.224, respectively, and the significance of miR-1306 was more obvious than TNM stage (Table 2).

Table 2 Multivariate Cox Regression Analysis to Evaluate the Prognostic Value of Patients' Clinical Features

\begin{tabular}{|l|c|c|c|}
\hline & HR Value & $\mathbf{9 5 \%} \mathbf{C I}$ & $\boldsymbol{P}$ value \\
\hline miR-1306 & 2.423 & $1.079-5.444$ & 0.032 \\
Age & 1.619 & $0.763-3.434$ & 0.209 \\
Gender & 1.173 & $0.57 \mathrm{I}-2.409$ & 0.663 \\
Smoking & 1.475 & $0.731-0.2 .974$ & 0.278 \\
LNM & 1.609 & $0.730-0.546$ & 0.238 \\
TNM stage & 2.224 & $1.060-4.666$ & 0.035 \\
Vascular invasion & $1.33 \mathrm{I}$ & $0.626-2.828$ & 0.457 \\
\hline
\end{tabular}

Abbreviation: LNM, lymph node metastasis.

\section{The Biological Effect of miR-I306 in LUSC}

Due to the relative high expression of miR-1306 in NCIH2170 and SK-MES-1 cells, these two cells were selected for further in vitro experiments. The expression level of miR-1306 in LUSC cells was regulated by the transfection of miR-1306 mimic and inhibitor. miR-1306 in was overexpressed by the transfection of mimic and silenced by inhibitor in NCI-H2170 (Figure 3A) and SK-MES-1 (Figure 3B, $P<0.01, P<0.001$ ).

The overexpression of miR-1306 exerted significantly promoted effects on the proliferation of NCI-H2170 and SK-MES-1 cells, which was suppressed by the knockdown of miR-1306 ( $P<0.05, P<0.01$, Figure 4A). While the migration (Figure 4B) and invasion (Figure 4C) of NCIH2170 and SK-MES-1 cells were accelerated by miR1306 and inhibited by the silencing of miR-1306 $(P<$ $0.01, P<0.001)$. 
A

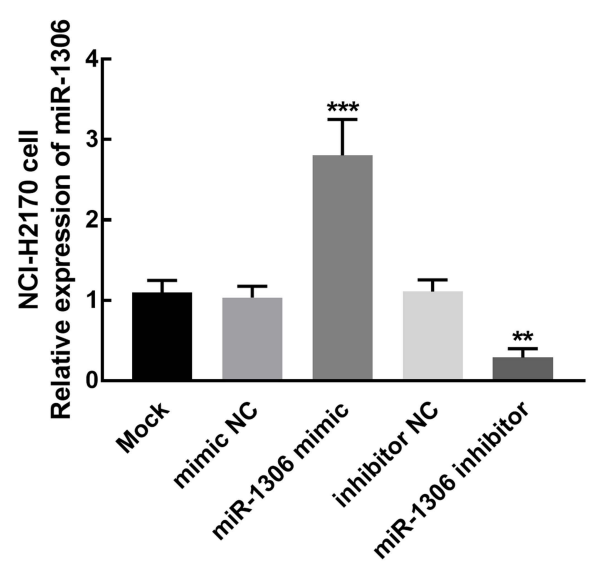

B

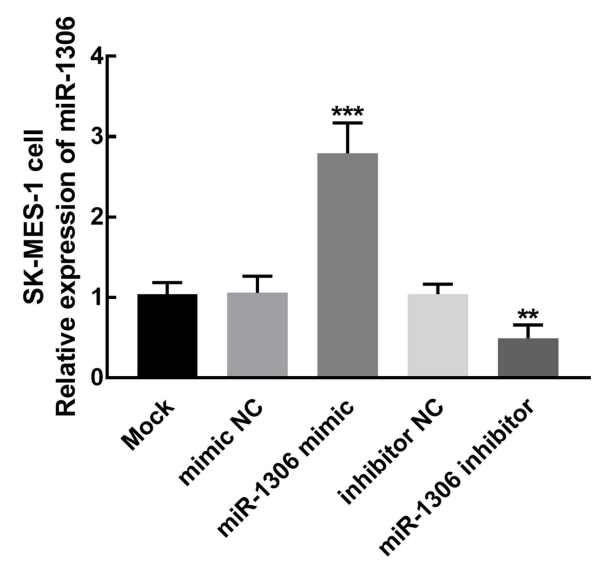

Figure 3 miR-I 306 was significantly overexpressed by the transfection of miR-I 306 mimic and suppressed by the transfection of miR-I306 inhibitor in $\mathrm{NCl}-\mathrm{H} 2 \mathrm{I} 70$ (A) and SK-MES-I (B) cells. $* * P<0.01$, $* * * P<0.00 I$.

A
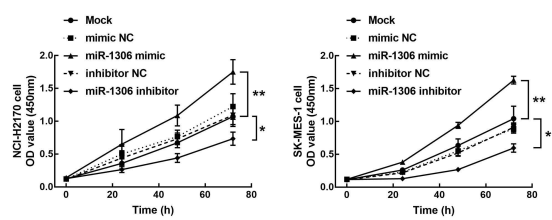

B

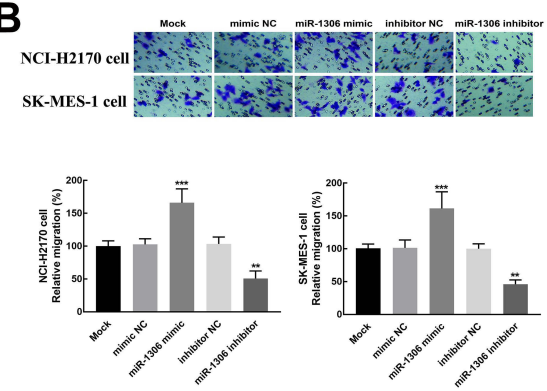

C
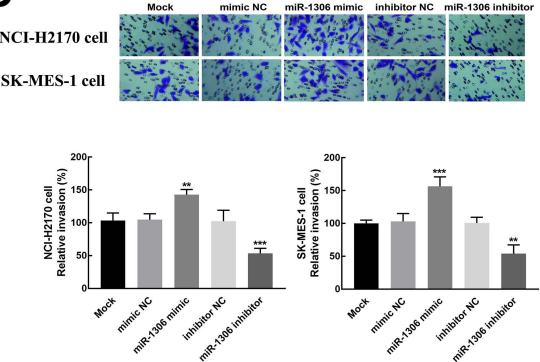

Figure 4 The biological effect of miR-I306 on LUSC cells. (A) The overexpression of miR-I306 significantly promoted the proliferation of NCl-2I70 and SK-MES-I cells. (B) The migration of $\mathrm{NCl}-2170$ and SK-MES-I cells was enhanced by the overexpression of miR-I306 and inhibited by the knockdown of miR-I306. (C) The invasion of $\mathrm{NCI}-2170$ and SK-MES-I cells was promoted by miR-I306 upregulation and suppressed by miR-I 306 silencing. $* P<0.05, * * P<0.01, * * * P<0.00 \mathrm{I}$.

\section{The Potential Mechanism Underlying miR-I306}

The binding sites between miR-1306 and RBM3 3'UTR (Figure 5A) were contained in the RBM3 wild-type vector (RBM3 WT). The relative luciferase activity of RBM3 WT was significantly suppressed by the overexpression of miR-1306 and enhanced by miR-1306 knockdown $(P<0.001$, Figure 5B). While the relative luciferase activity of the RBM3 mutant-type vector (RBM3 MT) was not affected by miR-1306 $(P>0.5$, Figure 5B).

\section{Discussion}

The initiation and development of LUSC is correlated with the various genetic and environmental factors. ${ }^{15}$ More and more investigations reported the expression profile of differently expressed miRNAs in human cancers, including LUSC. ${ }^{14,16}$ Dysregulated miRNAs are always considered as candidate biomarkers to screen the disease development of malignant tumors. For instance, the downregulation of miR-34c-3p could accelerate the migration and metastasis of NSCLC via upregulating integrin $\alpha 2 \beta 1 .{ }^{17}$ Previous bioinformatic analysis provides evidence for exploring the molecular mechanism underlying carcinogenesis. However, experimental validation and investigation of specific miRNAs are still needed. The significant upregulation of miR-1306 was observed in the present study, which is consistent with previous expression profile in NSCLC. ${ }^{14}$ The upregulation of miR-1306 was associated with the critical clinical features of LUSC patients, including the lymph node metastasis status and TNM stage. Lymph node metastasis and TNM stage of patients are two vital factors associated with the disease severity and development of patients. ${ }^{18,19}$ Therefore, miR-1306 was speculated to act as an indicator for the tumor progression of LUSC. Additionally, lymph node metastasis status and TNM stage are also important prognostic factors of LUSC patients. ${ }^{20,21}$ The significant relationship between miR-1306 expression 
A

RBM3 WT 5'...UCUUCAUCAGCUCUAGGAGGUGA...3' miR-1306 3'...ACCUGCAAACGUCCCCÚCCACC...5' RBM3 MT 5'...UCUUCAUCAGCUCUACCUCCACA...3'

B

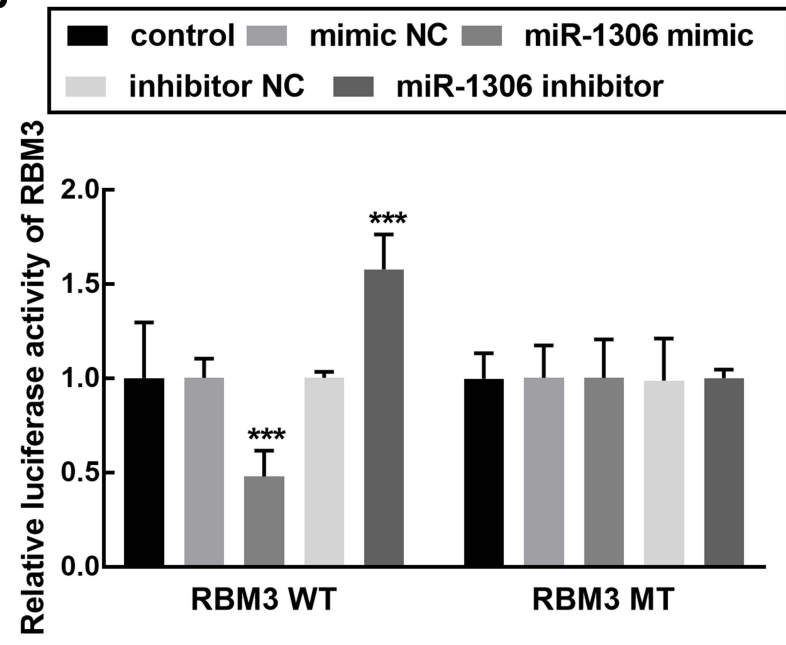

Figure 5 The mechanism underlying miR-1306 in LUSC. (A) Binding sites between miR-I306 and RBM3 WT and the mutations of RBM3 MT. (B) The overexpression of miR-1306 dramatically suppressed the luciferase activity of RBM3, which was enhanced by miR-1306 silencing. $* * * P<0.001$.

and these two clinical features also implying the potential prognostic significance of miR-1306 in LUSC. It was found that the upregulation of miR-1306 was dramatically correlated with the poor survival of LUSC patients, and miR1306 was also identified as an independent prognostic indicator of LUSC. Accordingly, miR-1306 was speculated to be a biomarker that could predict the disease development and clinical outcome of patients.

In addition, the functional role of miR-1306 in tumor cell growth, differentiation, and metastasis has also been reported in numerous cancers. For example, the downregulation of miR-1306 was negatively correlated with the prognosis of gastric cancer patients and reversed the promoted effect of circNHSL1 on tumor progression, indicating miR-1306 serve as a tumor suppressor. ${ }^{22}$ miRNAs always possess distinct expression in different cancers. Contrary to the function in gastric cancer, the tumor promoter role of miR-1306 was disclosed in hepatocellular carcinoma, where miR-1306 promoted the metastasis of hepatocellular carcinoma cells and epithelial-mesenchymal transition through regulating EBXL5. ${ }^{13}$ The biological function of miR-1306 has also been disclosed in other human diseases. For instance, upregulated miR-1306 could attenuate cell injury induced by ischemia/reperfusion and enhance cell survival rate via targeting BIK. ${ }^{12}$ miR-1306 could abrogate the inhibitory effect of lncRNA-SNHG7-003 on vascular smooth muscle cells growth and migration, which can serve as a therapeutic target of atherosclerosis. ${ }^{23}$ Here, miR-1306 was found to promote the proliferation, migration, and invasion of LUSC cells, suggesting its tumor promoter role in LUSC. Moreover, miR-1306 was found to regulate RBM3, which was reported to have significant prognostic significance in NSCLC. ${ }^{24}$ Therefore, miR-1306 was speculated to promote the progression of LUSC via modulating RBM3.

However, some limitations are still not negligible and need further investigations in this study. The relatively small sample size might limit the results to some degree. The results of Cox regression analysis only confirmed the prognostic value of miR-1306 and TNM stage. While the lymph node metastasis status of patients also plays an important role in the progression of LUSC, which was not shown in Cox regression analysis. ${ }^{20,25}$ Hence, a large sample size is needed in further studies.

\section{Conclusion}

Taken together, clinical findings revealed the prognostic value of miR-1306 in the development of LUSC, while the in vitro evidence demonstrated the tumor promoter role of miR-1306 in LUSC and the regulatory effect of miR-1306 on RBM3 was speculated to be the potential mechanism. The inhibition of miR-1306 might be a novel therapeutic strategy of LUSC.

\section{Ethics Statement}

This study had obtained approval from the Ethics Committee of Affiliated Hospital of Nantong University. The study was carried out after obtaining the written informed consent of all subjects.

\section{Funding}

This study was supported by Nantong Science and Technology Plan-New Key Research on Clinical Diagnosis and Treatment Technology (MS12018036).

\section{Disclosure}

The authors report no conflicts of interest in this work.

\section{References}

1. Herbst RS, Morgensztern D, Boshoff C. The biology and management of non-small cell lung cancer. Nature. 2018;553(7689):446-454. doi:10.1038/nature25183 
2. Torre LA, Siegel RL, Jemal A. Lung cancer statistics. Adv Exp Med Biol. 2016;893:1-19. doi:10.1007/978-3-319-24223-1_1

3. Yamamoto N, Yamanaka T, Ichinose Y, et al. Pooled analysis of S-1 trials in non-small cell lung cancer according to histological type. Anticancer Res. 2010;30(7):2985-2990.

4. Mairinger T. Histology, cytology and molecular diagnostics of lung cancer. Pathologe. 2019;40(6):649-661. doi:10.1007/s00292-01900677-8

5. Jones GS, Baldwin DR. Recent advances in the management of lung cancer. Clin Med. 2018;18(Suppl 2):s41-s46. doi:10.7861/clinmedicine.18-2-s41

6. Liu B, Li J, Cairns MJ. Identifying miRNAs, targets and functions. Brief Bioinform. 2014;15(1):1-19. doi:10.1093/bib/bbs075

7. Khan AQ, Ahmed EI, Elareer NR, Junejo K, Steinhoff M, Uddin S. Role of miRNA-regulated cancer stem cells in the pathogenesis of human malignancies. Cells. 2019;8(8). doi:10.3390/cells8080840

8. Iqbal MA, Arora S, Prakasam G, Calin GA, Syed MA. MicroRNA in lung cancer: role, mechanisms, pathways and therapeutic relevance. Mol Aspects Med. 2019;70:3-20. doi:10.1016/j.mam.2018.07.003

9. Yang S, Sui J, Liu T, et al. Expression of miR-486-5p and its significance in lung squamous cell carcinoma. $J$ Cell Biochem. 2019;120(8):13912-13923. doi:10.1002/jcb.28665

10. Filipska M, Skrzypski M, Czetyrbok K, et al. MiR-192 and miR-662 enhance chemoresistance and invasiveness of squamous cell lung carcinoma. Lung Cancer. 2018;118:111-118. doi:10.1016/j.lungcan. 2018.02.002

11. Walk EE, Yohe SL, Beckman A, et al. The cancer immunotherapy biomarker testing landscape. Arch Pathol Lab Med. 2020;144 (6):706-724. doi:10.5858/arpa.2018-0584-CP

12. Chen X, Li C, Li J, Sheng L, Liu X. Upregulation of miR-1306-5p decreases cerebral ischemia/reperfusion injury in vitro by targeting BIK. Biosci Biotechnol Biochem. 2019;83(12):2230-2237. doi:10. 1080/09168451.2019.1654846

13. He Z-J, Li W, Chen H, Wen J, Gao Y-F, Liu Y-J. miR-1306-3p targets FBXL5 to promote metastasis of hepatocellular carcinoma through suppressing snail degradation. Biochem Biophys Res Commun. 2018;504(4):820-826. doi:10.1016/j.bbrc.2018.09.059
14. Hu Y, Wang L, Gu J, Qu K, Wang Y. Identification of microRNA differentially expressed in three subtypes of non-small cell lung cancer and in silico functional analysis. Oncotarget. 2017;8 (43):74554-74566. doi:10.18632/oncotarget.20218

15. Nasim F, Sabath BF, Eapen GA. Lung cancer. Med Clin North Am. 2019;103(3):463-473. doi:10.1016/j.mcna.2018.12.006

16. Kulasingam V, Pavlou MP, Diamandis EP. Integrating high-throughput technologies in the quest for effective biomarkers for ovarian cancer. Nat Rev Cancer. 2010;10(5):371-378. doi:10.1038/nrc2831

17. Huang W, Yan Y, Liu Y, et al. Exosomes with low miR-34c-3p expression promote invasion and migration of non-small cell lung cancer by upregulating integrin $\alpha 2 \beta 1$. Signal Transduct Target Ther. 2020;5(1):39. doi:10.1038/s41392-020-0133-y

18. Akhurst T. Staging of non-small-cell lung cancer. PET Clin. 2018;13 (1):1-10. doi:10.1016/j.cpet.2017.09.004

19. Ustun F, Tokuc B, Tastekin E, Durmus Altun G. Tumor characteristics of lung cancer in predicting axillary lymph node metastases. Rev Esp Med Nucl Imagen Mol. 2019;38(2):80-86. doi:10.1016/j.remn.2018.09.010

20. Lu Y, Ma T, Wang L, Xue T. Advances in lymph node metastasis and lymph node dissection in early non-small cell lung cancer. Zhongguo Fei Ai Za Zhi. 2019;22(8):520-525. doi:10.3779/j.issn.1009-3419.2019.08.07

21. Woodard GA, Jones KD, Jablons DM. Lung cancer staging and prognosis. Cancer Treat Res. 2016;170:47-75.

22. Zhu Z, Rong Z, Luo Z, et al. Circular RNA circNHSL1 promotes gastric cancer progression through the miR-1306-3p/SIX1/vimentin axis. Mol Cancer. 2019;18(1):126. doi:10.1186/s12943-019-1054-7

23. Zheng J, Tan Q, Chen H, et al. IncRNA-SNHG7-003 inhibits the proliferation, migration and invasion of vascular smooth muscle cells by targeting the miR-1306-5p/SIRT7 signaling pathway. Int $J$ Mol Med. 2020;47(2):741-750. doi:10.3892/ijmm.2020.4821

24. Salomonsson A, Micke P, Mattsson JSM, et al. Comprehensive analysis of RNA binding motif protein 3 (RBM3) in non-small cell lung cancer. Cancer Med. 2020;9(15):5609-5619. doi:10.1002/cam4.3149

25. Kapoor C, Vaidya S, Wadhwan V, Malik S. Lymph node metastasis: a bearing on prognosis in squamous cell carcinoma. Indian J Cancer. 2015;52(3):417-424. doi:10.4103/0019-509X.176750

\section{Publish your work in this journal}

Cancer Management and Research is an international, peer-reviewed open access journal focusing on cancer research and the optimal use of preventative and integrated treatment interventions to achieve improved outcomes, enhanced survival and quality of life for the cancer patient.
The manuscript management system is completely online and includes a very quick and fair peer-review system, which is all easy to use. Visit http://www.dovepress.com/testimonials.php to read real quotes from published authors. 